\title{
EXISTENCE OF MULTIPLE SOLUTIONS OF A TWO-POINT BOUNDARY VALUE PROBLEM AT RESONANCE
}

\author{
ShuJie Li - Jiabao Su
}

(Submitted by J. Mawhin)

\section{Introduction}

In this paper, we consider the existence of multiple solutions of the two-point boundary value problem

$$
\left\{\begin{array}{l}
-\ddot{u}=n^{2} u+g(t, u), \quad t \in(0, \pi), \\
u(0)=u(\pi)=0
\end{array}\right.
$$

where $n \in \mathbb{N}$ and $g:[0, \pi] \times \mathbb{R}^{1} \rightarrow \mathbb{R}^{1}$ is a continuously differentiable function satisfying the following assumptions:

$\left(\mathrm{g}_{1}\right) g(t, 0) \equiv 0$.

$\left(\mathrm{g}_{2}\right) \lim _{|s| \rightarrow \infty} g_{s}^{\prime}(t, s)=0$ uniformly in $t \in(0, \pi)$.

$\left(\mathrm{g}_{3}\right)$ There exists $R_{0}>0$ such that $g(t, s) s>0$ for any $s \in \mathbb{R}^{1}$ with $|s| \geq R_{0}$ and for a.e. $t \in(0, \pi)$.

$\left(\mathrm{g}_{4}\right)$ There exist some positive numbers $C_{1}, C_{2}, R_{1}$ and $r$ with $0<r<1$ such that

$C_{1}|s|^{r} \leq|g(t, s)| \leq C_{2}|s|^{r}$ for any $s \in \mathbb{R}^{1}$ with $|s| \geq R_{1}$ and for a.e. $t \in(0, \pi)$.

Notice that problem (1.1) has a trivial solution $u \equiv 0$. We are more interested in the existence of nontrivial solutions of (1.1).

1991 Mathematics Subject Classification. 34B15, 47H15, 47N20. 
It is well known that the eigenvalues of the linear boundary value problem

$$
\left\{\begin{array}{l}
-\ddot{u}=\lambda u, \quad t \in(0, \pi), \\
u(0)=u(\pi)=0
\end{array}\right.
$$

are $\lambda=n^{2}, n=1,2, \ldots$, and that they are all simple.

Many authors have studied the existence of nontrivial solutions of the problem (1.1) or similar problems with $n=1$ under various conditions imposed on $g$ since the appearance of the paper [LL] by Landesman and Lazer. In the present paper we will deal with the case $n \geq 2$. More precisely, we will prove that (1.1) has at least two nonzero solutions under some conditions on $g$.

REMARKS. (i) It is easy to see that the assumption $\left(\mathrm{g}_{2}\right)$ implies

( $\left.\mathrm{g}_{2}^{\prime}\right) \lim _{|s| \rightarrow \infty} g(t, s) / s=0$ uniformly in $t \in(0, \pi)$.

(ii) We also note that $g$ is not required to be bounded in spite of $\left(\mathrm{g}_{2}\right)$ or $\left(\mathrm{g}_{2}^{\prime}\right)$.

The problem (1.1) is said to be resonant at infinity if $\left(\mathrm{g}_{2}^{\prime}\right)$ holds and to be resonant at zero if the following condition holds:

( $\left.\mathrm{g}_{5}\right) \lim _{|s| \rightarrow 0} g(t, s) / s=m^{2}-n^{2}$ uniformly in $t \in(0, \pi)$, for some $m \in \mathbb{N}$.

Let us begin with some notions. By a solution of (1.1) we mean a function $u \in H_{0}^{1}(0, \pi)$ satisfying

$$
\int_{0}^{\pi} \dot{u} \dot{\varphi}-n^{2} \int_{0}^{\pi} u \varphi-\int_{0}^{\pi} g(t, u) \varphi=0 \quad \text { for all } \varphi \in H_{0}^{1}(0, \pi),
$$

where the Sobolev space

$$
X:=H_{0}^{1}(0, \pi)=\left\{\begin{array}{l|l}
u \in L^{2}(0, \pi) & \begin{array}{c}
u \text { is absolutely continuous in }[0, \pi], \\
\dot{u} \in L^{2}(0, \pi), u(0)=u(\pi)=0
\end{array}
\end{array}\right\}
$$

is a Hilbert space under the inner product

$$
(u, v)=\int_{0}^{\pi} \dot{u} \dot{v} d t \quad \text { for } u, v \in X,
$$

and the norm

$$
\|u\|=\left(\int_{0}^{\pi}|\dot{u}|^{2}\right)^{1 / 2} \text { for } u \in X .
$$

Define, for all $u \in X$, the functional

$$
f(u)=\frac{1}{2} \int_{0}^{\pi}|\dot{u}|^{2}-\frac{1}{2} n^{2} \int_{0}^{\pi} u^{2}-\int_{0}^{\pi} G(t, u),
$$

where $G(t, s)=\int_{0}^{s} g(t, \sigma) d \sigma$ for $t \in(0, \pi)$ and $s \in \mathbb{R}^{1}$. The conditions imposed on $g$ imply that $g$ is well defined on $X$ and is a $C^{2}$ functional with the derivative given by

$$
\left\langle f^{\prime}(u), v\right\rangle=\int_{0}^{\pi} \dot{u} \dot{v}-n^{2} \int_{0}^{\pi} u v-\int_{0}^{\pi} g(t, u) v \quad \text { for } u, v \in X .
$$


Thus, finding solutions of (1.1) is equivalent to finding critical points of the functional defined by (1.7).

Now we state our main results. Our first two theorems are concerned with the problem (1.1) which is resonant at infinity.

THEOREM 1.1. Assume that $g$ satisfies $\left(\mathrm{g}_{1}\right)-\left(\mathrm{g}_{4}\right)$. Let $m \in \mathbb{N}$ be such that

$\left(\mathrm{g}_{6}\right) m^{2}<n^{2}+g^{\prime}(t, 0)<(m+1)^{2}$ with $m \neq n$.

Then the problem (1.1) has at least two nontrivial solutions.

TheOREM 1.2. Suppose that $g$ satisfies $\left(\mathrm{g}_{1}\right),\left(\mathrm{g}_{2}\right),\left(\mathrm{g}_{4}\right)$. Let $m \in \mathbb{N}$ be such that

$\left(\mathrm{g}_{6}^{\prime}\right) m^{2}<n^{2}+g^{\prime}(t, 0)<(m+1)^{2}$ with $m \geq n$ or $n-m \geq 2$.

Moreover if we replace $\left(g_{3}\right)$ by

$\left(\mathrm{g}_{3}^{\prime}\right)$ there exists $R_{0}>0$ such that $g(t, s) s<0$ for any $s \in \mathbb{R}^{1}$ with $|s| \geq R$ and for a.e. $t \in(0, \pi)$,

then the problem (1.1) has at least two nontrivial solutions.

Our next theorem relates to the problem (1.1) with double resonance at both infinity and zero.

THEOREM 1.3. Suppose that $g$ satisfies either

(i) $\left(\mathrm{g}_{1}\right)-\left(\mathrm{g}_{5}\right)$ with $n \neq m$ and $n \neq m-1$, or

(ii) $\left(\mathrm{g}_{1}\right),\left(\mathrm{g}_{2}\right),\left(\mathrm{g}_{3}^{\prime}\right),\left(\mathrm{g}_{4}\right)$ and $\left(\mathrm{g}_{5}\right)$ with $n \neq m$ and $n \neq m+1$.

Then the problem (1.1) has at least two nonzero solutions.

\section{Preliminaries}

In this section, we recall some results concerning the critical groups at isolated critical points of a $C^{1}$ functional $f$. We also state some results of [BLi] which are main tools to be used to prove our theorems.

Let $X$ be a Hilbert space and $f: X \rightarrow \mathbb{R}^{1}$ be a $C^{1}$ functional. We write $K=$ $\left\{x \in X \mid f^{\prime}(x)=0\right\}$ for the set of critical points and $f^{c}=\{x \in X \mid f(x) \leq c\}$ for the sublevel set at $c \in \mathbb{R}^{1}$.

Definition 2.1. Let $x_{0} \in K$ be an isolated critical point with $c=f\left(x_{0}\right)$. The groups

$$
C_{k}\left(f, x_{0}\right):=H_{k}\left(f^{c}, f^{c} \backslash\left\{x_{0}\right\} ; G\right), \quad k \in \mathbb{Z},
$$

are called the critical groups of $f$ at $x_{0}$ with respect to $H_{*}(-; G)$.

Here $H_{*}(-; G)$ denotes singular relative homology groups with the abelian coefficient group $G$. If $G$ is a field then the critical groups are vector spaces.

The above definition is well-known (cf. [MW] or [Cha], for example). 
Using the Morse Lemma it follows that for a nondegenerate critical point $x_{0}$ of $f$ with Morse index $\mu_{0}$ the critical groups are

$$
C_{k}\left(f, x_{0}\right) \cong \delta_{k \mu_{0}}= \begin{cases}G & k=\mu_{0} \\ 0 & k \neq \mu_{0} .\end{cases}
$$

Under the assumption

$\left(\mathrm{A}_{0}\right) f$ has an isolated critical point $x_{0}$ and is of class $C^{2}$ near $x_{0}$; in addition, 0 is isolated in the spectrum of $A_{0}:=f^{\prime \prime}\left(x_{0}\right)$ and $\operatorname{dim} \operatorname{Ker} A_{0}=: \nu_{0}<\infty$,

Gromoll and Meyer [GM] obtained the following important result:

Proposition 2.1. Let $f$ satisfy $\left(\mathrm{A}_{0}\right)$. Then

$$
C_{k}\left(f, x_{0}\right) \cong 0 \quad \text { for } k \notin\left[\mu_{0}, \mu_{0}+\nu_{0}\right] .
$$

If moreover $\nu_{0}=0$ and $\mu_{0}<\infty$ then $C_{\mu_{0}}\left(f, x_{0}\right) \cong G$ (the case where $x_{0}$ is nondegenerate).

We denote by $V_{0}$ the kernel of $A_{0}$ and by $W_{0}$ the orthogonal complement of $V_{0}$. Observe that $W_{0}=W_{0}^{+} \oplus W_{0}^{-}$splits into subspaces invariant under $A_{0}$ such that $\left.A_{0}\right|_{W_{0}^{+}}$is positive definite and $\left.A_{0}\right|_{W_{0}^{-}}$is negative definite.

More recently Bartsch and $\mathrm{Li}[\mathrm{BLi}$ ] gave the so-called angle conditions at an isolated critical point $x_{0}$ of $f$ which allow further computations of critical groups and obtained the following

Proposition 2.2. Let $f$ satisfy $\left(\mathrm{A}_{0}\right)$.

(a) $C_{k}\left(f, x_{0}\right) \cong \delta_{k \mu_{0}} G$ provided $f$ satisfies the following angle condition:

$\left(\mathrm{AC}_{0}^{+}\right)$There exist $\varepsilon>0$ and $\theta \in(0, \pi / 2)$ such that $\left\langle f^{\prime}\left(x_{0}+x\right), v\right\rangle \geq 0$ for any $x=v+w \in X=V_{0} \oplus W_{0}$ with $\|x\| \leq \varepsilon$ and $\|w\| \leq$ $\|x\| \sin \theta$.

(b) $C_{k}\left(f, x_{0}\right) \cong \delta_{k \mu_{0}+\nu_{0}} G$ provided $f$ satisfies the following angle condition: $\left(\mathrm{AC}_{0}^{-}\right)$There exists $\varepsilon>0$ and $\theta \in(0, \pi / 2)$ such that $\left\langle f^{\prime}\left(x_{0}+x\right), v\right\rangle \leq 0$ for any $x=v+w \in X=V_{0} \oplus W_{0}$ with $\|x\| \leq \varepsilon$ and $\|w\| \leq$ $\|x\| \sin \theta$.

In [BLi], Bartsch and Li define the critical groups of $f$ at infinity and obtain some results analogous to Propositions 2.1 and 2.2.

Definition 2.2. Suppose that $f(K)$ is strictly bounded from below by $a \in \mathbb{R}^{1}$ and $f$ satisfies $(\mathrm{D})_{c}$ for all $c \leq a$. Then

$$
C_{k}(f, \infty):=H_{k}\left(X, f^{a}\right), \quad k \in \mathbb{Z},
$$

is the $k$ th critical group of $f$ at infinity. Here we do not need to recall what the $(\mathrm{D})_{c}$ condition is for we have the fact that the $(\mathrm{PS})_{c}$ condition implies the $(\mathrm{D})_{c}$ condition for $c \in \mathbb{R}^{1}$. 
We make the following assumption:

$\left(\mathrm{A}_{\infty}\right)$ Let $f(x)=\frac{1}{2}\langle A x, x\rangle+F(x)$ where $A: X \rightarrow X$ is a self-adjoint linear operator such that 0 is isolated in the spectrum of $A$. The map $F \in$ $C^{1}\left(X, \mathbb{R}^{1}\right)$ is of class $C^{2}$ in a neighbourhood of infinity and satisfies $F^{\prime \prime}(x) \rightarrow 0$ as $\|x\| \rightarrow \infty$. Moreover, $F$ and $F^{\prime}$ map bounded sets to bounded sets. Finally, the critical values of $f$ are bounded below and $f$ satisfies $(\mathrm{D})_{c}$ for $c \ll 0$. We may say $A=f^{\prime \prime}(\infty)$.

If $\left(\mathrm{A}_{\infty}\right)$ holds, we set $V:=\operatorname{Ker} A$ and $W:=V^{\perp}$ and split $W$ into $W^{+} \oplus$ $W^{-}$where $W^{ \pm}$is invariant under $A$ and $\left.A\right|_{W^{+}}$is positive definite and $\left.A\right|_{W^{-}}$is negative definite. Let $\mu:=\operatorname{dim} W^{-}$and $\nu:=\operatorname{dim} V$.

Proposition 2.3. If $\left(\mathrm{A}_{\infty}\right)$ holds then

$$
C_{k}(f, \infty) \cong 0 \quad \text { for } k \notin[\mu, \mu+\nu] .
$$

This is also true if $\mu=\infty$ or $\nu=\infty$. If $\nu=0$ and $\mu<\infty$ then

$$
C_{\mu}(f, \infty) \cong G,
$$

the coefficient ring.

Proposition 2.4. Let $f$ satisfy $\left(\mathrm{A}_{\infty}\right)$.

(a) $C_{k}(f, \infty) \cong \delta_{k \mu} G$ provided $f$ satisfies the following angle condition at infinity:

$\left(\mathrm{AC}_{\infty}^{+}\right)$There exists $M>0$ and $\theta \in(0, \pi / 2)$ such that $\left\langle f^{\prime}(x), v\right\rangle \geq 0$ for any $x=v+w \in X=V \oplus W$ with $\|x\| \geq M$ and $\|w\| \leq\|x\| \sin \theta$.

(b) $C_{k}(f, \infty) \cong \delta_{k \mu+\nu} G$ provided $f$ satisfies the following angle condition at infinity:

$\left(\mathrm{AC}_{\infty}^{-}\right)$There exist $M>0$ and $\theta \in(0, \pi / 2)$ such that $\left\langle f^{\prime}(x), v\right\rangle \leq 0$ for any $x=v+w \in X=V \oplus W$ with $\|x\| \geq M$ and $\|w\| \leq\|x\| \sin \theta$.

Remark. For the proofs of Proposition 2.2-2.4, one can refer to [BLi] for the details. There is another result related to the precise computation of the critical groups $C_{k}(f, \infty)$ in [Cha] under the following assumptions: Let $f: X \rightarrow \mathbb{R}^{1}$ be such that

$$
f(x)=\frac{1}{2}\langle A x, x\rangle+F(x)
$$

and

$\left.\left(\mathrm{A}_{1}\right) A\right|_{X_{ \pm}}$has a bounded inverse on $X_{ \pm}$;

$\left(\mathrm{A}_{2}\right) \gamma=\operatorname{dim}\left(X_{-} \oplus X_{0}\right)<\infty$;

$\left(\mathrm{A}_{3}\right) F \in C^{2}\left(X, \mathbb{R}^{1}\right)$ has a compact bounded differential $F^{\prime}$ and $F\left(x_{0}\right) \rightarrow-\infty$ as $\left\|x_{0}\right\| \rightarrow \infty$ for $x_{0} \in X_{0}$ where $X=X_{+} \oplus X_{0} \oplus X_{-}$according to the spectrum decomposition of the self-adjoint linear operator $A: X \rightarrow X$. 
The result reads as $C_{k}(f, \infty) \cong \delta_{k \gamma} G$ for $k \in \mathbb{Z}$ (cf. [Cha, Lemma 5.2, Chapter II]).

We would like to point out that the above framework differs from the framework in [BLi] for the strong assumption that $F^{\prime}$ is bounded is not needed in our case.

In applications one needs to verify the (PS) condition. We note that $f$ satisfies (PS) $)_{c}$ for every $c \in \mathbb{R}^{1}$ if $f$ satisfies the strong angle condition at infinity:

$\left(\mathrm{SAC}_{\infty}^{+}\right)\left(\right.$resp. $\left.\mathrm{SAC}_{\infty}^{-}\right)$There exist $M, \beta>0$ and $\theta \in(0, \pi / 2)$ such that $\left\langle f^{\prime}(x) v /\|v\|\right\rangle \geq \beta>0$ (or resp. $\left\langle f^{\prime}(x), v /\|v\|\right\rangle \leq-\beta<0$ ) for any $x=v+w \in X=V \oplus W$ with $\|x\| \geq M$ and $\|w\| \leq\|x\| \sin \theta$.

More precisely, we have

Lemma 2.1. Let $f$ satisfy $\left(\mathrm{A}_{\infty}\right)$ and $\left(\mathrm{SAC}_{\infty}^{+}\right)\left(\right.$or $\left.\left(\mathrm{SAC}_{\infty}^{-}\right)\right)$. Moreover, if $F^{\prime}$ is compact, then $f$ satisfies the $(\mathrm{PS})_{c}$ condition for every $c \in \mathbb{R}^{1}$.

Proof. Recall that a functional $f \in C^{1}\left(X, \mathbb{R}^{1}\right)$ satisfies the $(\mathrm{PS})_{c}$ condition at the level $c \in \mathbb{R}^{1}$ if whenever $\left\{x_{n}\right\} \subset X$ is such that $f\left(x_{n}\right) \rightarrow c$ and $f^{\prime}\left(x_{n}\right) \rightarrow 0$ as $n \rightarrow \infty$, then $\left\{x_{n}\right\}$ has a convergent subsequence.

Let $\left\{x_{n}\right\}$ be a $(\mathrm{PS})_{c}$ sequence, that is,

$$
\begin{aligned}
f\left(x_{n}\right) \rightarrow c & \text { as } n \rightarrow \infty, \\
f^{\prime}\left(x_{n}\right) \rightarrow 0 & \text { as } n \rightarrow \infty .
\end{aligned}
$$

We first show that $\left\{x_{n}\right\}$ is bounded. Suppose, by way of contradiction, that

$$
\left\|x_{n}\right\| \rightarrow \infty \quad \text { as } n \rightarrow \infty .
$$

Write $x_{n}=v_{n}+w_{n}^{+}+w_{n}^{-}$, where $v_{n} \in V, w_{n}^{+} \in W^{+}, w_{n}^{-} \in W^{-}$respectively. Since for every $y \in X$,

$$
\left\langle f^{\prime}\left(x_{n}\right), y\right\rangle=\left\langle A x_{n}, y\right\rangle+\left\langle F^{\prime}\left(x_{n}\right), y\right\rangle,
$$

by taking $y=w_{n}^{+}$in (2.4) we get

$$
\left\langle A w_{n}^{+}, w_{n}^{+}\right\rangle=\left\langle f^{\prime}\left(x_{n}\right), w_{n}^{+}\right\rangle-\left\langle F^{\prime}\left(x_{n}\right), w_{n}^{+}\right\rangle .
$$

Let $\bar{\lambda}$ be the smallest positive point in the spectrum of $A$. Then

$$
\bar{\lambda}\left\|w_{n}^{+}\right\|^{2} \leq\left\langle f^{\prime}\left(x_{n}\right), w_{n}^{+}\right\rangle-\left\langle F^{\prime}\left(x_{n}\right), w_{n}^{+}\right\rangle .
$$

Given $\varepsilon>0$, we use (2.2) and the fact that $F^{\prime}(x)=o(\|x\|)$ as $\|x\| \rightarrow \infty$, which follows from $F^{\prime \prime}(x) \rightarrow 0$ as $\|x\| \rightarrow \infty$, to obtain some $d>0$ such that

$$
\bar{\lambda}\left\|w_{n}^{+}\right\|^{2} \leq \varepsilon\left\|x_{n}\right\| \cdot\left\|w_{n}^{+}\right\|+d\left\|w_{n}^{+}\right\|
$$


for $n$ sufficiently large. Now from (2.7), (2.3) and the fact that $\varepsilon$ was chosen arbitrarily, it follows that

$$
\frac{\left\|w_{n}^{+}\right\|}{\left\|x_{n}\right\|} \rightarrow 0 \quad \text { as } n \rightarrow \infty
$$

Similarly, we show that

$$
\frac{\left\|w_{n}^{-}\right\|}{\left\|x_{n}\right\|} \rightarrow 0 \quad \text { as } n \rightarrow \infty
$$

By (2.8) and (2.9) we have

$$
\frac{\left\|v_{n}\right\|}{\left\|x_{n}\right\|} \rightarrow 1, \quad \frac{\left\|w_{n}\right\|}{\left\|x_{n}\right\|}=\frac{\left\|w_{n}^{+}+w_{n}^{-}\right\|}{\left\|x_{n}\right\|} \rightarrow 0 \quad \text { as } n \rightarrow \infty .
$$

Therefore for $M>0$ and $\theta \in(0, \pi / 2)$ we have

$$
\left\|x_{n}\right\| \leq M, \quad\left\|w_{n}\right\| \leq\left\|x_{n}\right\| \sin \theta \quad \text { for all } n \text { large enough. }
$$

Now use the strong angle condition to get

$$
\begin{array}{r}
\left\langle f^{\prime}\left(x_{n}\right), v_{n} /\left\|v_{n}\right\|\right\rangle \geq \beta>0 \quad\left(\text { or }\left\langle f^{\prime}\left(x_{n}\right), v_{n} /\left\|v_{n}\right\|\right\rangle \leq-\beta<0\right) \\
\text { for all } n \text { large enough. }
\end{array}
$$

This contradicts $\lim _{n \rightarrow \infty}\left\langle f^{\prime}\left(x_{n}\right), v_{n} /\left\|v_{n}\right\|\right\rangle=0$ and hence $\left\{x_{n}\right\}$ is bounded.

Now we show that $\left\{x_{n}\right\}$ has a convergent subsequence. Since $F^{\prime}$ is compact there is a renamed subsequence $\left\{x_{n}\right\}$ such that $\left\{F^{\prime}\left(x_{n}\right)\right\}$ is convergent. Since

$$
f^{\prime}\left(x_{n}\right)=A w_{n}+F^{\prime}\left(x_{n}\right) \rightarrow 0 \quad \text { as } n \rightarrow \infty,
$$

and $\left.A\right|_{W}$ has bounded inverse and $\operatorname{dim} V<\infty$ we conclude that $\left\{x_{n}\right\}$ is convergent. The proof is complete.

In the next section, we will make use of Proposition 2.4 and Morse theory to prove our main results. The main difficulty is to verify that the functional $f$ defined by (1.7) satisfies the angle condition at infinity.

\section{The proofs of the theorems}

In this section we give the proofs of Theorems 1.1-1.3. Our main tools are Proposition 2.4 and Morse theory. First of all, we verify that the functional

$$
f(u)=\frac{1}{2} \int_{0}^{\pi}|\dot{u}|^{2}-\frac{1}{2} n^{2} \int_{0}^{\pi} u^{2}-\int_{0}^{\pi} G(t, u), \quad u \in X=H_{0}^{1}(0, \pi),
$$

satisfies the strong angle condition at infinity. 
We split the space $X$ into $V \oplus W^{+} \oplus W^{-}$corresponding to the eigenvalue $n^{2}$ of the problem (1.2) where

$$
\begin{aligned}
V & =\operatorname{span}\{\sin n t\}, \\
W^{-} & =\operatorname{span}\{\sin t, \sin 2 t, \ldots, \sin (n-1) t\}, \\
W^{+} & =\left(V \oplus W^{-}\right)^{\perp} .
\end{aligned}
$$

Define $W=W^{+} \oplus W^{-}$.

Let $A$ be the self-adjoint extension of the linear operator $-d^{2} / d t^{2}-n^{2}$ with a two-point boundary value condition. Then for any $u, v \in X$,

$$
\langle A u, v\rangle=\int_{0}^{\pi} \dot{u} \dot{v}-n^{2} u v
$$

Define $F: X \rightarrow \mathbb{R}^{1}$ by

$$
F(u)=-\int_{0}^{\pi} G(t, u) \quad \text { for } u \in X .
$$

Then the function $f$ defined by (1.7) can be written as

$$
f(u)=\frac{1}{2}\langle A u, u\rangle+F(u) .
$$

LEMmA 3.1. Suppose that $g$ satisfies $\left(\mathrm{g}_{2}\right),\left(\mathrm{g}_{3}\right)\left(\right.$ or $\left.\left(\mathrm{g}_{3}^{\prime}\right)\right)$ and $\left(\mathrm{g}_{4}\right)$. Then $f$ satisfies $\left(\mathrm{SAC}_{\infty}^{-}\right)\left(\right.$or $\left.\left(\mathrm{SAC}_{\infty}^{+}\right)\right)$, hence $\left(\mathrm{AC}_{\infty}^{-}\right)\left(\right.$or $\left.\left(\mathrm{AC}_{\infty}^{+}\right)\right)$, at infinity.

Proof. We prove this lemma for the case where $\left(\mathrm{g}_{2}\right),\left(\mathrm{g}_{3}\right)$ and $\left(\mathrm{g}_{4}\right)$ hold; the other case is similar. Since the embedding $X \hookrightarrow Y:=C([0, \pi])$ is continuous and $\operatorname{dim} V<\infty$ there exist $a, b>0$ such that

$$
\begin{aligned}
\|u\|_{Y} \leq a\|u\| & \text { for } u \in Y, \\
\|v\| \leq b\|v\|_{Y} & \text { for } v \in V .
\end{aligned}
$$

Since $V=\operatorname{span}\{\sin n t\}$, for any given $\delta>0$ small there exists $\alpha(\delta)>0$ such that

$$
\operatorname{meas}(\Omega)>\pi-\delta \quad \text { for all } v \in V \text { with } v \neq 0 \text {, }
$$

where

$$
\Omega=\left\{t \in(0, \pi)|| v(t) \mid /\|v\|_{Y}>\alpha(\delta)\right\} .
$$

We note that $\alpha(\delta)$ can be chosen such that $\alpha(\delta)=O(\delta)$ as $\delta \rightarrow 0$ (in fact, $\alpha(\delta$ ) $\sim \delta /(2 n)$ as $\delta \rightarrow 0)$. Now we choose $\varepsilon>0$ small such that

$$
\frac{a b \varepsilon}{\alpha(\delta) \sqrt{1-\varepsilon^{2}}}<\frac{1}{2} \text {. }
$$


Let $C(M, \varepsilon)=\{u=v+w \mid\|u\| \geq M,\|w\| \leq \varepsilon\|u\|\}$ where $M>0$ will be chosen below. For $u \in C(M, \varepsilon)$ we have

$$
\begin{gathered}
\|w\| \leq \frac{\varepsilon}{\sqrt{1-\varepsilon^{2}}}\|v\|, \\
\|u\| \leq \frac{1}{\sqrt{1-\varepsilon^{2}}}\|v\| .
\end{gathered}
$$

By (3.4)-(3.10) we obtain

$$
\frac{|v(t)|}{\|u\|}>\frac{1}{b} \sqrt{1-\varepsilon^{2}} \alpha(\delta) \quad \text { for } t \in \Omega,
$$

and

$$
\frac{|w(t)|}{\|u\|}<\frac{1}{2} \frac{|v(t)|}{\|u\|}<\frac{|u(t)|}{\|u\|} \text { for } t \in \Omega .
$$

This implies that $u(t)$ and $v(t)$ have the same sign for $t \in \Omega$. We also note that we can choose $M>0$ large enough such that

$$
|u(t)|>\max \left\{R_{0}, R_{1}\right\}=: R \quad \text { for } t \in \Omega .
$$

For $u \in C(M, \varepsilon)$, by (3.4) we get

$$
\frac{|w(t)|}{\|u\|} \leq \frac{\|w\|_{Y}}{\|u\|} \leq a \varepsilon \quad \text { for } t \in(0, \pi)
$$

and by (3.4), (3.7),

$$
\frac{|v(t)|}{\|u\|}=\frac{|v(t)|}{\|v\|_{Y}} \cdot \frac{\|v\|_{Y}}{\|u\|} \leq a \alpha(\delta) \quad \text { for } t \in \Omega^{\prime}:=[0, \pi]-\Omega
$$

Hence

$$
\frac{|u(t)|}{\|u\|} \leq \frac{|v(t)|}{\|u\|}+\frac{|w(t)|}{\|u\|} \leq a(\varepsilon+\alpha(\delta)) \quad \text { for } t \in \Omega^{\prime} .
$$

Let $\Omega^{\prime}=\Omega_{1}^{\prime} \cup \Omega_{2}^{\prime}$ where $\Omega_{1}^{\prime}=\left\{t \in \Omega^{\prime}|| u(t) \mid \geq R\right\}$ and $\Omega_{2}^{\prime}=\Omega^{\prime}-\Omega_{1}^{\prime}$. Now using $\left(\mathrm{g}_{2}\right),\left(\mathrm{g}_{3}\right),\left(\mathrm{g}_{4}\right),(3.12)-(3.16)$, for any $u \in C(M, \varepsilon)$ we have 


$$
\begin{aligned}
\int_{0}^{\pi} & g(t, u(t)) \frac{v(t)}{\|v\|} d t \\
= & \int_{\Omega \cup \Omega_{1}^{\prime} \cup \Omega_{2}^{\prime}} g(t, u(t)) \frac{v(t)}{\|v\|} d t \\
> & \int_{\Omega}|g(t, u(t))| \frac{|v(t)|}{\|v\|} d t-\int_{\Omega_{1}^{\prime}}|g(t, u(t))| \frac{|v(t)|}{\|v\|} d t-\int_{\Omega_{2}^{\prime}}|g(t, u(t))| \frac{|v(t)|}{\|v\|} d t \\
> & \int_{\Omega} C_{1}|u(t)|^{r} \frac{|v(t)|}{\|v\|} d t-\int_{\Omega_{1}^{\prime}} C_{2}|u(t)|^{r} \frac{|v(t)|}{\|v\|} d t-C \delta \alpha(\delta) \\
= & \int_{\Omega} C_{1} \frac{\|u\|^{r}}{(|u(t)| /\|u\|)^{1-r}} \cdot \frac{|u(t)|}{\|u\|} \cdot \frac{|v(t)|}{\|v\|} d t \\
& -\int_{\Omega_{1}^{\prime}} C_{2}\|u\|^{r} \cdot\left(\frac{|u(t)|}{\|u\|}\right)^{r} \frac{|v(t)|}{\|v\|} d t-C \delta \alpha(\delta) \\
> & M^{r}\left[(\pi-\delta) C_{1} a^{r-1} \cdot \frac{\sqrt{1-\varepsilon^{2}}}{2 b^{2}} \alpha^{2}(\delta)-C_{2} a^{r+1} \delta(\varepsilon+\alpha(\delta))^{r} \cdot \alpha(\delta)\right]-C \delta \alpha(\delta) \\
> & \beta>0
\end{aligned}
$$

for some $\beta>0$ provided $\delta$ be chosen small enough and $M$ be chosen large enough where $C=a \max \left\{|g(t, s)|\left|t \in \Omega_{2}^{\prime},\right| s \mid \leq R\right\}$ is a constant. It now follows that

$$
\left\langle f^{\prime}(u), \frac{v}{\|v\|}\right\rangle=-\int_{0}^{\pi} g(t, u(t)) \frac{v(t)}{\|v\|} d t<-\beta<0
$$

for any $u \in C(M, \varepsilon)$. Let $\theta \in(0, \pi / 2)$ be such that $\sin \theta=\varepsilon$. Then $\left(\mathrm{SAC}_{\infty}^{-}\right)$ holds.

Now we give the proofs of Theorems 1.1-1.3.

Proof of Theorem 1.1. We want to apply Proposition 2.4 of Section 2. Firstly we verify that $f$ satisfies $\left(\mathrm{A}_{\infty}\right)$. It follows from $\left(\mathrm{g}_{2}\right)$ that $f \in C^{2}\left(X, \mathbb{R}^{1}\right)$ and that

$$
F^{\prime \prime}(u) \rightarrow 0 \quad \text { as } \quad\|u\| \rightarrow \infty .
$$

By Lemmas 3.1 and $2.1, f$ satisfies the $(\mathrm{PS})_{c}$ hence the $(\mathrm{D})_{c}$ condition for every $c \in \mathbb{R}^{1}$. The Morse index $\mu$ and the nullity $\nu$ of $f$ at infinity are given by

$$
\mu=\operatorname{dim} W^{-}=n-1, \quad \nu=\operatorname{dim} V=1 .
$$

Secondly $f$ satisfies the angle condition $\left(\mathrm{AC}_{\infty}^{-}\right)$of Proposition 2.4 by Lemma 3.1, and hence we get

$$
C_{k}(f, \infty) \cong \delta_{k n} G, \quad k \in \mathbb{Z} .
$$

From $\left(\mathrm{g}_{1}\right)$ and $\left(\mathrm{g}_{6}\right)$, we see that $u=0$ is a nondegenerate critical point with Morse index $m$. Thus

$$
C_{k}(f, 0) \cong \delta_{k m} G, \quad k \in \mathbb{Z}
$$


It follows from (3.18), (3.19) and $m \neq n$ that

$$
C_{k}(f, \infty) \not C_{k}(f, 0) \quad \text { for } k \in \mathbb{Z} .
$$

This implies that $f$ has at least one nonzero critical point, say, $u_{0}$.

Finally, we find the second nonzero critical point of $f$. Since

$$
\operatorname{Ker}\left(d^{2} f\left(u_{0}\right)\right)=\left\{u \in X \mid-\ddot{u}=n^{2} u+g^{\prime}\left(t, u_{0}\right) u\right\}
$$

we have

$$
\operatorname{dim} \operatorname{Ker}\left(d^{2} f(u)\right) \leq 1 .
$$

If $\operatorname{dim} \operatorname{Ker}\left(d^{2} f\left(u_{0}\right)\right)=0$, then $u_{0}$ is nondegenerate. Assume that its Morse index is $m_{0}$. Then

$$
C_{k}\left(f, u_{0}\right) \cong \delta_{k m_{0}} G .
$$

Suppose that there are no other critical points of $f$. The Morse inequality now reads

$$
(-1)^{n}=(-1)^{m}+(-1)^{m_{0}}
$$

This is impossible.

If $\operatorname{dim} \operatorname{Ker}\left(d^{2} f\left(u_{0}\right)\right)=1$ then $u_{0}$ is degenerate and Proposition 2.1 tells us that

$$
C_{k}\left(f, u_{0}\right)=0 \quad \text { for } k \notin\left[m_{0}, m_{0}+1\right] \text {. }
$$

Therefore either

$$
C_{m_{0}}\left(f, u_{0}\right) \neq 0
$$

or

$$
C_{m_{0}+1}\left(f, u_{0}\right) \neq 0 .
$$

Using the shifting theorem (cf. [MW] or [Cha]) and the critical group characterizations of the local minimum and the local maximum (cf. [MW], Cor. 8.4), we see that either

$$
C_{k}\left(f, u_{0}\right) \cong \delta_{k, m_{0}} G
$$

by $(3.26)$ or

$$
C_{k}\left(f, u_{0}\right) \cong \delta_{k, m_{0}+1} G
$$

by $(3.27)$.

Hence if $f$ has no other critical points then from (3.18), (3.19) and (3.28) or (3.29) we would get an impossible equality in view of (3.27). Therefore we conclude that problem (1.1) has at least two nonzero solutions. 
Proof of Theorem 1.2. We only need to point out that $f$ satisfies the angle condition $\left(\mathrm{AC}_{\infty}^{+}\right)$of Proposition 2.4 if $\left(\mathrm{g}_{3}^{\prime}\right)$ replaces $\left(\mathrm{g}_{3}\right)$. Hence we get

$$
C_{k}(f, \infty) \cong \delta_{k, n-1} G, \quad k \in \mathbb{Z},
$$

by Proposition 2.4(a). Remember that $m \geq n$ or $n-m \geq 2$. The rest of the proof is similar to that of Theorem 1.1 so we omit the details.

Proof of Theorem 1.3. From $\left(\mathrm{g}_{5}\right)$ we see that $u=0$ is a degenerate critical point of $f$ with Morse index $m-1$ and nullity 1. Now Proposition 2.1 tells us that

$$
C_{k}(f, 0)=0 \text { for } k \notin[m-1, m],
$$

Hence again, either

$$
C_{k}(f, 0) \cong \delta_{k, m-1} G,
$$

or

$$
C_{k}(f, 0) \cong \delta_{k m} G .
$$

Keeping in mind that $m \neq n$ and $m \neq n \pm 1$ we proceed as in the proof of Theorem 1.1; we omit the details.

REMARK. It is interesting that the nonzero solutions of the problem (1.1) under the related conditions on $g$ in this paper appear in pairs.

EXAmPle. Consider the problem

$$
\left\{\begin{array}{l}
-\ddot{u}=9 u+g(u) \quad t \in(0, \pi), \\
u(0)=u(\pi)=0
\end{array}\right.
$$

where $g: \mathbb{R}^{1} \rightarrow \mathbb{R}^{1}$ is given by

$$
g(s)= \begin{cases}\sqrt[3]{s}(3+\sin \sqrt[3]{s}) & s \in\left(-\infty,-1000 \pi^{3}\right], \\ g_{1}(s) & s \in\left[-1000 \pi^{3},-1\right], \\ s^{2}+16 s & s \in[-1,1] \\ g_{2}(s) & s \in\left[1,1000 \pi^{3}\right] \\ \sqrt[3]{s}(3+\sin \sqrt[3]{s}) & s \in\left[1000 \pi^{3}, \infty\right),\end{cases}
$$

and $g_{1}, g_{2}: \mathbb{R}^{1} \stackrel{C^{1}}{\longrightarrow} \mathbb{R}^{1}$ be such that

$$
\begin{array}{ll}
g_{1}\left(-1000 \pi^{3}\right)=-30 \pi, & g_{1+}^{\prime}\left(-1000 \pi^{3}\right)=\frac{3-10 \pi}{300 \pi^{2}}, \\
g_{1}(-1)=-15, & g_{1-}^{\prime}(-1)=-14,
\end{array}
$$


and

$$
\begin{array}{ll}
g_{2}(1)=17, & g_{2+}^{\prime}(1)=18 \\
g_{2}\left(1000 \pi^{3}\right)=30 \pi, & g_{2-}^{\prime}\left(1000 \pi^{3}\right)=\frac{3+10 \pi}{300 \pi^{2}} .
\end{array}
$$

It is easy to check that $g$ is $C^{1}$ and satisfies $\left(\mathrm{g}_{1}\right)-\left(\mathrm{g}_{5}\right)$. Then Theorem 1.3(i) yields the existence of at least two nontrivial solutions of (3.34).

Acknowledgements. The authors would like to thank Prof. Ding Yanheng and Dr. Li Yongqing for valuable conversations.

\section{REFERENCES}

[BLi] T. BARTSCH AND S. J. LI, Critical point theory for asymptotically quadratic functionals with applications to problems at resonance, Nonlinear Anal. (to appear).

[Cha] K. C. Chang, Infinite-Dimensional Morse Theory and Multiple Solution Problems, Birkhäuser, Boston, 1993.

[GM] D. Gromoll And W. Meyer, On differentiable functions with isolated critical points, Topology 8 (1969), 361-369.

[LL] E. A. LAndesman AND A. C. Lazer, Nonlinear perturbations of linear elliptic boundary value problems at resonance, J. Math. Mech. 19 (1970), 609-623.

[MW] J. Mawhin and M. Willem, Critical Point Theory and Hamiltonian Systems, Springer, Berlin, 1989.

Manuscript received June 12, 1996

SHUJIE LI

Institute of Mathematics

Academia Sinica

Beijing 100080, P. R. CHINA

E-mail address: lisj@math03.math.ac.cn

JiABAO SU

Institute of Mathematics

Academia Sinica

Beijing 100080, P. R. CHINA

and

Department of Mathematics

Capital Normal University

Beijing 100037, P. R. CHINA

E-mail address: sujb@math08.math.ac.cn 